\title{
Household Food Security among Different Wealth Groups within Uyo Metropolis in Southern Nigeria
}

\author{
Opara Dominica $\mathbf{C}^{1}$, Johnson Ofonime $\mathbf{E}^{2 \text {,* }}$ \\ ${ }^{1}$ Department of Community Health, University of Uyo, Uyo, Nigeria \\ ${ }^{2}$ Department of Community Health, University of Uyo/ University of Uyo Teaching Hospital, Uyo, Nigeria \\ *Corresponding author: drjohnsonoe@yahoo.com
}

Received November 17, 2018; Revised December 27, 2018; Accepted January 06, 2019

\begin{abstract}
Studies done in various states in Nigeria have shown alarming rates of food insecurity among the population. The aim of this study was to determine the prevalence of household food insecurity among different wealth groups in Uyo metropolis, in southern Nigeria and to determine compensatory feeding patterns engaged in by studied food insecure households. This was a cross sectional descriptive study carried out among 249 households of different wealth groups based on the occupational status and educational attainment of the household heads. Data was collected using interviewer administered semi-structured questionnaire and analyzed using SPSS version 20 with a 0.05 level of significance. The prevalence of food insecurity among surveyed households was $89.9 \%$ with food insecure 26.5\% (without hunger), 30.5\% (moderate hunger) and $32.9 \%$ (severe hunger). Marital status, socioeconomic class, household size and household income all had statistically significant relationships with food security status $(\mathrm{p}<0.05)$. Those in socioeconomic class- 1 were the most food secure $(23.8 \%)$, while $63.6 \%$, and $36.4 \%$ of households in socioeconomic class-5 were food insecure with moderate hunger and severe hunger respectively. Up to $36.4 \%$ of those with a household size of $>7$ suffered food insecurity with severe hunger. Regarding household income, $48.9 \%$ of households with income less than 50,000 naira per month, were food insecure with severe hunger $(\mathrm{p}<0.05)$. Coping strategies engaged in by food insecure households included borrowing money from friends, $33.7 \%$, collecting food from friends $26.1 \%$ and sending children to work, $8.8 \%$. There was high prevalence of food insecurity among studied households. It is recommended that better educational opportunities be made available to those of the low socioeconomic class, so as to ensure gainful employment. Minimum wage in Nigeria should be increased to ensure increased household income.
\end{abstract}

Keywords: household food security, household food insecurity, wealth groups, household income, coping strategies

Cite This Article: Opara DC, and Johnson OE, "Household Food Security among Different Wealth Groups within Uyo Metropolis in Southern Nigeria." Journal of Food Security, vol. 7, no. 1 (2019): 1-7. doi: $10.12691 /$ jfs-7-1-1.

\section{Introduction}

Food insecurity is a great challenge to the policy makers and the world nations due to the huge increase in the human population across the whole world. People are said to be food secure when at all times, physical, social and economic access to sufficient, safe and nutritious food is available and meets their dietary needs and food preferences for an active and healthy life [1,2]. Access, food availability, utilization and stability underpin food security coupled by the nutrition security achieved for a household when secure access to food are available with a sanitary environment, adequate health services, and adequate care $[3,4]$.

Food and Agriculture Organization (FAO) estimates that over 800 million $(10.9 \%)$ of the world's population currently suffer from chronic under-nutrition. Food insecurity is closely linked with hunger and poverty; and over 700 million people live under conditions of extreme poverty i.e., earning less than US\$1 per day $[5,6,7,8]$. A number of factors such as income, gender, educational level of heads of households, geographical location and household sizes are known to affect household food security, as they directly affect economic access and the sustenance of such access $[9,10]$.

The reduction of global hunger and food insecurity observed between 1990 and 2014 has witnessed an increase over the last three years [5]. In the West African region, though progress has been made, there is still a large proportion of the population who suffer food insecurity. This is because the rate of population growth in the region far exceeds the quantity and quality of food available to feed the populace [11].

Negligence of the agricultural sector in favour of the petroleum industry has led Nigeria to a high reliance on food imports. Many hitherto food secure households have therefore been thrown into vulnerability due to high prices of staple foods, high fuel price, increased cost of 
transportation, mass layoffs, high unemployment rate etc.; all consequences of the economic recession caused by dwindling oil prices and falling Naira to Dollar rate. It is well established that economic vulnerability is inversely related to food security [12]. There is high amount of food wastage yearly during the harvest season due to poor storage and transportation facilities, reducing food availability in the country [13].

Nigeria is included among countries faced with serious food insecurity problems and on the 2017 Global Hunger Index Nigeria was ranked $84^{\text {th }}$ out of 119 countries $[14,15]$. Studies in various states in Nigeria have reported alarming rates of food insecurity among the populace $[14,16,17]$.

Hunger affects virtually every aspect of life. Household consequences of food insecurity can therefore be grouped into physical, psychological and socio-familial $[18,19]$. Not only does food insecurity in itself have deleterious effects, but compensatory efforts may also exact a heavy toll on households. To cope with food insecurity, households may feed the children first at the expense of the nutrient needs of adults, reduce food consumption, or sell productive assets $[3,20,21]$.

In Akwa Ibom state, rapid urbanization has led to widespread changes in land use giving rise to problems like flooding and depletion of the nutritional value of the soil which have contributed to food insecurity in the state [22]. A study of food security and quality of life among students in the University of Uyo in 2016 revealed that $84 \%$ of students were food insecure, while among rural farming households, high levels of household food security was reported $[10,23]$. However, there is not much of information on the food security status of households in urban centers, particularly Uyo with the presumption that unlike rural farming households, they need to buy most if not all the food consumed. Also with the rather high level of food insecurity reported among University of Uyo students in a previous study, it is imperative to investigate the household food security outlook in the homes. This study therefore set out to measure household food security status across different wealth groups and to determine compensatory feeding patterns engaged in by food insecure households in Uyo, Akwa Ibom State.

\section{Materials and Method}

Study Site: The study was carried out in Uyo, the capital of Akwa Ibom state in the South-South region of Nigeria. The population of Uyo, according to the 2016 estimated Nigerian Census was 429,900 [24]. The inhabitants of Uyo work in both the formal and informal sectors.

Study design: This was a cross sectional descriptive study.

\subsection{Inclusion Criteria}

Only members of the household that had correct knowledge and were involved in the purchase, preparation and feeding of the household were included in the study. Hence, the respondents were either the heads of the households or their spouses.

\subsection{Sample Size}

A sample size of 192 was derived using the formula:

$$
n=z p q / d^{2}
$$

Where $\mathrm{z}=95 \%$; confidence interval $=1.96$.

$\mathrm{p}=$ probability of event occurring, (taken as 0.436 , as reported household that were food insecure without hunger in a previous study [14].

$$
q=1-p=1-0.436=0.564
$$

$\mathrm{d}=0.05$ which is acceptable margin of error.

Therefore $\mathrm{n}=192$.

This was increased to 249 to take care of attrition, non - response and incomplete information.

\subsection{Sampling Technique}

Cluster sampling method was employed in the selection of different households. Different clusters were identified where it was assumed that different wealth groups may be found (including unemployed persons, traders ,artisans, motor park attendants, taxi/bus drivers and state and federal civil servants, owners of small and large business premises.) The locations included 2 major motor parks in Uyo namely Akwa Ibom Transport Company (AKTC) park and Lagos park at Itam; the two major local markets namely Akpan Andem market and Itam market; the state and federal secretariats and the mechanic village on Abak road. At each of the locations, alternate consenting participants who met the inclusion criteria were enrolled in the study.

\subsection{Data Collection/Instrument of Data Collection}

Data was collected by 2 previously trained graduates of sociology who served as research assistants. Data collection lasted for 5 days.A structured questionnaire was administered to each of the 249 respondents. It had four sections; Section A: Socio-demographic characteristics of the respondents such as gender, marital status, highest educational level attained, occupational status, household size and household income.

Section B: Household food security; this study used the USDA approach for the analysis of household food security in the selected study area. This approach groups households with the aid of a constructed food security scale that contains 18 questions for households with children and 10 questions for households without children. Each of these questions has three responses namely; often true and sometimes true which were considered as affirmative responses and were given a score of 1 ; and never true which was given a score of 0 . The household food security status was categorized into four groups which were scored as follows:

1. Food secure households: 0-2 for households with and without children;

2. Food insecure without hunger households: 3-7 for households with children and 3-5 for households without children; 
3. Food insecure with moderate hunger household: 8-12 for households with children and 6-8 for households without children; and

4. Food insecure with severe hunger households: 13-18 for households with children and 9-10 for households without children.

Section C: Household socio-economic class. This was determined using a modification of the method of Oyedeji, 1985 in the selected study area. This index is based on the occupational status and education attained by the household heads. For occupation, class 1 was allocated to senior public servants, professionals, managers, large scale traders, business men and contractors; class 2 to intermediate grade public servants and school teachers; class 3 to drivers and artisans; class 4 to petty traders, laborers, messengers and class 5 to unemployed, full time housewives, students and subsistence farmers. For educational scale, class 1 was awarded to university graduates or equivalents, class 2 to school certificate holders (ordinary level GCE) who also had teacher or other professional training; class 3 to school certificate or grade II teachers' certificate holders or equivalent; class 4 to those who had modern three and primary 6 certificates and 5 to those who had no formal education. The mean of the two scores to the nearest whole number was the socioeconomic class assigned to the household heads, with class 1 being the highest and class 5 being the lowest socioeconomic class.

Section D: This section assessed the compensatory feeding pattern engaged in by food insecure households, such as borrowing money from friends, collecting food from friends or sending children to beg.

\subsection{Statistical Analysis}

The data collected was analyzed using Statistical Package for Social Sciences (SPSS), version 20.0 (SPSS Inc. Chicago, IL, USA). The categorical variable results were presented as frequency and its percentage. Inferential statistics was done using chi square and Spearman's correlation coefficient to establish associations and relationships. Level of significance was set at $\mathrm{p}<0.05$.

\subsection{Ethical Approval}

Ethical approval for the study was obtained from Ministry of Health, Akwa Ibom State. In addition the purpose of the study was explained to prospective respondents, the confidentiality of the information they were to provide assured and they were not required to supply their names. They were free to opt out. Only those that gave consent were recruited as respondents.

\section{Results}

\subsection{Socio-demographic Characteristics of Respondents}

One hundred and thirty out of 249 respondents were females; $47.0 \%$ were aged 36-50 years and majority, $81.1 \%$ were married. The highest proportion of household heads $(55.0 \%)$ had a monthly income of less than fifty thousand naira, with $88(35.3 \%)$ having a household size of 5-7 members. Up to $30.5 \%$ of the households belonged to class 4 socioeconomic class. (Table 1)

Table 1. Socio-demographic characteristics of respondents

\begin{tabular}{|c|c|c|}
\hline Variable & $\begin{array}{c}\text { Frequency } \\
\mathrm{N}=249\end{array}$ & Percentage \\
\hline Age & & \\
\hline $20-35$ & 31 & 12.5 \\
\hline $36-50$ & 117 & 47.0 \\
\hline $51-65$ & 92 & 36.9 \\
\hline$>65$ & 9 & 3.6 \\
\hline Sex & & \\
\hline Male & 119 & 47.8 \\
\hline Female & 130 & 52.2 \\
\hline Marital status & & \\
\hline Single & 14 & 5.6 \\
\hline Married & 202 & 81.1 \\
\hline Divorced & 19 & 7.6 \\
\hline Widowed & 14 & 5.6 \\
\hline Socio-economic class & & \\
\hline Class 1 & 21 & 8.4 \\
\hline Class 2 & 73 & 29.3 \\
\hline Class 3 & 57 & 22.9 \\
\hline Class 4 & 76 & 30.5 \\
\hline Class 5 & 22 & 8.9 \\
\hline Household size & & \\
\hline $1-4$ & 139 & 55.8 \\
\hline $5-7$ & 88 & 35.3 \\
\hline$>7$ & 22 & 8.8 \\
\hline Household income (Naira) & & \\
\hline$<50,000$ & 137 & 55.0 \\
\hline $50,000-100,000$ & 98 & 39.4 \\
\hline$>100,000$ & 14 & 3.6 \\
\hline
\end{tabular}

\subsection{Relationship between Selected Socio-demographic Characteristics of Respondents and Household Food Security}

There was a statistically significant association between food security and marital status, social class, household size and income $(\mathrm{p}<0.05)$. Only $25(10.1 \%)$ of respondents were food secure

Overall, $82(32.9 \%)$ of the households were food insecure with severe hunger, while $66(26.5 \%)$ and 76 $(30.5 \%)$ were food insecure without hunger and with moderate hunger respectively. With respect to marital status, $50 \%$ of single respondents were food secure, while $57.2 \%$ of widowed households were food insecure with severe hunger.

Socioeconomic class had a statistically significant relationship with food security status. Those in socioeconomic class 1 , were the most food secure $(23.8 \%)$ followed by class $2(19.2 \%)$. No social class 5 household was food secure. However, $63.6 \%$, and $36.4 \%$ of socioeconomic class 5 were food insecure with moderate hunger and severe hunger respectively, while $56.6 \%$ of socioeconomic class 4 were food insecure with severe hunger $(\mathrm{p}<0.05)$.

Up to $36.4 \%$ of those with a household size of $>7$ suffered food insecurity with severe hunger.

Regarding household income, $48.9 \%$ of households with income less than 50,000 naira per month, were food 
insecure with severe hunger, while $34.7 \%$ of those who earned between 50,000-100,000 naira per month had food insecurity with moderate hunger. (Table 2)

\subsection{Coping Strategies of Respondents}

About a third of the households (33.7\%) reported that borrowing money from friends was the most frequently used coping strategy. More than $26 \%$ of respondents reported collecting food from friends, while $22(8.8 \%)$ sent their children to work. Other coping strategies reported by respondents included starving themselves to feed the children $(6.0 \%)$ and sending children to stay in a relative's/friend's house $(4.0 \%)$. The highest proportion of households who borrowed money $(100 \%)$, collected food from friends $(40.9 \%)$ and sent children to work $(22.7 \%)$ belonged to social class 5 . Households that sent children to work commonly earned $<50,000$ naira $(\mathrm{p}<0.05)$. (Table 3 ).

Table 2. Association between selected socio-demographic characteristics of household heads and household food security status

\begin{tabular}{|c|c|c|c|c|c|}
\hline \multirow{2}{*}{ Variable } & \multirow{2}{*}{ Food secure } & \multicolumn{3}{|c|}{ Food insecure } & \multirow{2}{*}{$\begin{array}{l}\text { Test statistics; } \\
\text { p value }\end{array}$} \\
\hline & & Without hunger & With moderate hunger & With severe hunger & \\
\hline \multicolumn{6}{|l|}{ Marital status } \\
\hline Single & $7(50.0)$ & $5(35.7)$ & $1(7.15)$ & $1(7.15)$ & \multirow{4}{*}{$\begin{array}{l}x^{2}=39.2 \\
p=0.00 *\end{array}$} \\
\hline Married & $14(6.9)$ & $53(26.2)$ & $65(32.2)$ & $70(34.7)$ & \\
\hline Divorced & $2(10.5)$ & $5(26.3)$ & $9(47.4)$ & $3(15.8)$ & \\
\hline Widowed & $2(14.3)$ & $3(21.4)$ & $1(7.1)$ & $8(57.2)$ & \\
\hline \multicolumn{5}{|l|}{ Socio-economic class } & \multirow{6}{*}{$\begin{array}{l}\mathrm{r}=0.522 \\
\mathrm{p}=0.000^{*}\end{array}$} \\
\hline Class 1 & $5(23.8)$ & $9(42.9)$ & $5(23.8)$ & $2(9.5)$ & \\
\hline Class 2 & $14(19.2)$ & $37(50.7)$ & $20(27.4)$ & $2(2.7)$ & \\
\hline Class 3 & $3(5.3)$ & $16(28.1)$ & $11(19.3)$ & $27(47.3)$ & \\
\hline Class 4 & $3(3.9)$ & $4(5.3)$ & $26(34.2)$ & $43(56.6)$ & \\
\hline Class 5 & $0(0.0)$ & $0(0.0)$ & $14(63.6)$ & $8(36.4)$ & \\
\hline \multicolumn{5}{|l|}{ Household size } & \multirow{4}{*}{$\begin{array}{c}x^{2}=23.7 \\
p=0.001^{*}\end{array}$} \\
\hline $1-4$ & $18(12.9)$ & $24(17.3)$ & $51(36.7)$ & $46(33.1)$ & \\
\hline $5-7$ & $3(3.4)$ & $33(37.5)$ & $24(27.3)$ & $28(31.8)$ & \\
\hline$>7$ & $4(18.2)$ & $9(40.9)$ & $1(4.5)$ & $8(36.4)$ & \\
\hline \multicolumn{5}{|l|}{ Household income } & \multirow{5}{*}{$\begin{array}{c}\mathrm{r}=-0.310 \\
\mathrm{p}=0.000^{*}\end{array}$} \\
\hline$<50,000$ & $10(7.3)$ & $19(13.9)$ & $41(29.9)$ & $67(48.9)$ & \\
\hline $50,000-100,000$ & $10(10.2)$ & $42(42.9)$ & $34(34.7)$ & $12(12.2)$ & \\
\hline$>100,000$ & $5(35.7)$ & $5(35.7)$ & $1(7.2)$ & $3(21.4)$ & \\
\hline Total & $25(10.1)$ & $66(26.5)$ & $76(30.5)$ & $82(32.9)$ & \\
\hline
\end{tabular}

$*=$ statistically significant $(\mathrm{p}<0.05)$.

Table 3. Association between selected socio-demographic characteristics of household heads and coping strategies

\begin{tabular}{|c|c|c|c|c|}
\hline & $\begin{array}{c}\text { Borrow money from friends. } \\
\mathrm{N}(\%)\end{array}$ & $\begin{array}{l}\text { Collect food from friends. } \\
\mathrm{N}(\%)\end{array}$ & $\begin{array}{c}\text { Send children to work. N } \\
(\%)\end{array}$ & $\begin{array}{l}\text { Others } \\
\mathrm{N}(\%) \\
\end{array}$ \\
\hline \multicolumn{5}{|l|}{ Marital status } \\
\hline Single & 11(78.6) & $0(0.0)$ & $0(0.0)$ & $0(0.0)$ \\
\hline Married & $66(32.7)$ & $60(29.7)$ & $19(9.4)$ & $25(69.4)$ \\
\hline Divorced & $5(26.3)$ & $3(15.8)$ & $1(5.3)$ & $7(19.4)$ \\
\hline Widowed & $2(14.3)$ & $2(14.3)$ & $2(14.3)$ & $4(11.1)$ \\
\hline Statistics; $p$ value & $\mathrm{x}^{2}=14.93 ; \mathrm{p}=0.02 *$ & $\mathrm{x}^{2}=12.76 ; \mathrm{p}=0.01^{*}$ & $x^{2}=1.58 ; p=0.67$ & $x^{2}=7.55 ; 0.11$ \\
\hline \multicolumn{5}{|l|}{ Socio-economic class } \\
\hline Class 1 & $0(0.0)$ & $6(28.6)$ & $0(0.0)$ & $4(11.1)$ \\
\hline Class 2 & $19(26.0)$ & $31(42.5)$ & $0(0.0)$ & $7(19.4)$ \\
\hline Class 3 & $21(36.8)$ & $1(1.8)$ & $7(12.3)$ & $6(16.7)$ \\
\hline Class 4 & $22(28.9)$ & $20(26.3)$ & $11(14.5)$ & $15(41.7)$ \\
\hline Class 5 & $22(100.0)$ & $9(40.9)$ & $5(22.7)$ & $4(11.1)$ \\
\hline Statistics; $p$ value & $\mathrm{x}^{2}=10.31 ; \mathrm{p}=0.24$ & $\mathrm{x}^{2}=18.74 ; \mathrm{p}=0.00^{*}$ & $\mathrm{x}^{2}=23.47 ; \mathrm{p}=0.00^{*}$ & $\mathrm{x}^{2}=13.77 ; \mathrm{p}=0.09$ \\
\hline \multicolumn{5}{|l|}{ Household size } \\
\hline $1-4$ & $48(35.3)$ & $40(28.8)$ & $12(8.6)$ & $16(44.4)$ \\
\hline $5-7$ & $27(30.7)$ & $21(23.8)$ & $8(9.1)$ & $18(50.0)$ \\
\hline$>7$ & $9(40.9)$ & $4(18.2)$ & $2(9.1)$ & $2(5.6)$ \\
\hline Statistics; $p$ value & $\mathrm{x}^{2}=1.12 ; \mathrm{p}=0.89$ & $\mathrm{x}^{2}=2.14 ; \mathrm{p}=0.34$ & $\mathrm{x}^{2}=0.1 .64 ; \mathrm{p}=0.41$ & $x^{2}=1.64 ; p=0.41$ \\
\hline \multicolumn{5}{|l|}{ Household income } \\
\hline below 50,000 & $49(35.8)$ & $34(24.8)$ & $21(15.3)$ & $20(55.6)$ \\
\hline $50,000-100,000$ & $31(31.6)$ & $31(31.6)$ & $0(0.0)$ & $14(38.9)$ \\
\hline$>100,000$ & $4(28.6)$ & $0(0.0)$ & $1(7.1)$ & $2(5.6)$ \\
\hline Statistics; $p$ value & $x^{2}=3.57 ; p=0.74$ & $\mathrm{x}^{2}=8.45 ; \mathrm{p}=0.08$ & $\mathrm{x}^{2}=24.36 ; \mathrm{p}=0.00^{*}$ & $\mathrm{x}^{2}=8.14 ; \mathrm{p}=0.23$ \\
\hline Total & 84 & 65 & 22 & 36 \\
\hline
\end{tabular}

$*=$ statistically significant $(\mathrm{p}<0.05)$.

$\mathrm{x}^{2}=$ Likelihood ratio chi square 


\subsection{Relationship between Selected} Socio-demographic Characteristics of Households and Different Food Security Measures

Socioeconomic class and household income were seen to have significant relationships with all the measures of food security. Worrying about food running out, giving low cost food to family members and children not eating enough were all significantly associated with low social class and low income (Table 4). Low household income and low social class were also significantly associated with skipping of meals by adult household members, weight loss due to insufficient food (Table 5), children skipping meals or reducing size of meals (Table 6).

Table 4. Distribution of food security measures of households by selected socio-demographic characteristics

\begin{tabular}{|c|c|c|c|c|c|c|}
\hline Variable & $\begin{array}{c}\text { A (variable; } \\
\text { statistic) }\end{array}$ & $\begin{array}{c}\text { B (variable; } \\
\text { statistic) }\end{array}$ & $\begin{array}{l}\text { C( variable; } \\
\text { statistic) }\end{array}$ & $\begin{array}{l}\text { D ( variable; } \\
\text { statistic) }\end{array}$ & $\begin{array}{l}\text { E ( variable; } \\
\text { statistic) }\end{array}$ & $\begin{array}{c}\text { F ( variable; } \\
\text { statistic) }\end{array}$ \\
\hline $\begin{array}{l}\text { Marital status } \\
\text { Single } \\
\text { Married } \\
\text { Divorced } \\
\text { Widowed }\end{array}$ & $\begin{array}{c}\mathrm{r}=-0.098 \\
\mathrm{p}=0.123\end{array}$ & $\begin{array}{l}\mathrm{r}=0.063 ; \\
\mathrm{p}=0.319\end{array}$ & $\begin{array}{l}r=0.156 \\
p=0.013\end{array}$ & $\begin{array}{c}\mathrm{r}=-0.035 \\
\mathrm{p}=0.586\end{array}$ & $\mathrm{r}=0.073 ; \mathrm{p}=0.261$ & $\begin{array}{c}\mathrm{r}=-0.042 \\
\mathrm{p}=0.518\end{array}$ \\
\hline $\begin{array}{l}\text { Socio-economic } \\
\text { Class } 1 \\
\text { Class } 2 \\
\text { Class } 3 \\
\text { Class } 4 \\
\text { Class } 5\end{array}$ & $\begin{array}{l}\mathrm{r}=-0.298 \\
\mathrm{p}=0.000 *\end{array}$ & $\begin{array}{l}\mathrm{r}=0.292 \\
\mathrm{p}=0.000 *\end{array}$ & $\begin{array}{l}\mathrm{r}=0.458 \\
\mathrm{p}=0.000^{*}\end{array}$ & $\begin{array}{l}r=-0.478 \\
p=0.000 *\end{array}$ & $\begin{array}{l}\mathrm{r}=0.258 \\
\mathrm{p}=0.000^{*}\end{array}$ & $\begin{array}{l}\mathrm{r}=-0.454 \\
\mathrm{p}=0.000 *\end{array}$ \\
\hline $\begin{array}{l}\text { Household size } \\
1-4 \\
5-7 \\
>7\end{array}$ & $\begin{array}{l}r=0.047 \\
p=0.461\end{array}$ & $\begin{array}{c}\mathrm{r}=-0.110 \\
\mathrm{p}=0.083\end{array}$ & $\begin{array}{c}\mathrm{r}=-0.111 \\
\mathrm{p}=0.081\end{array}$ & $\begin{array}{l}\mathrm{r}=0.129 \\
\mathrm{p}=0.047 *\end{array}$ & $\mathrm{r}=0.024 ; \mathrm{p}=0.713$ & $\begin{array}{l}\mathrm{r}=0.079 \\
\mathrm{p}=0.225\end{array}$ \\
\hline $\begin{array}{l}\text { Household inco } \\
>50,000 \\
50,000-100,000 \\
>100,000\end{array}$ & $\begin{array}{l}\mathrm{r}=0.222 \\
\mathrm{p}=0.000 *\end{array}$ & $\begin{array}{l}\mathrm{r}=-0.162 ; \\
\mathrm{p}=0.010 *\end{array}$ & $\begin{array}{l}r=-0.247 \\
p=0.000 *\end{array}$ & $\begin{array}{l}\mathrm{r}=0.254 \\
\mathrm{p}=0.000 *\end{array}$ & $\begin{array}{l}\mathrm{r}=-0.202 \\
\mathrm{p}=0.002 *\end{array}$ & $\begin{array}{l}\mathrm{r}=0.213 ; \\
\mathrm{p}=0.001 *\end{array}$ \\
\hline
\end{tabular}

*statistically significant $(\mathrm{p}<0.05)$

$\mathrm{A}=$ worry food will run out; $\mathrm{B}=$ Have enough resource for food; $\mathrm{C}=\mathrm{Can}$ afford balanced meals; $\mathrm{D}=$ give children low cost food; $\mathrm{E}=$ can afford balanced meal for children; $\mathrm{F}=$ children not eating enough can't afford it.

Table 5. Distribution of food security measures of households by selected socio-demographic characteristics contd

\begin{tabular}{|c|c|c|c|c|c|}
\hline Variable & $\begin{array}{l}\text { G (variable; } \\
\text { statistic) }\end{array}$ & $\begin{array}{l}\text { H (variable; } \\
\text { statistic) }\end{array}$ & I (variable; statistic) & $\begin{array}{c}\mathbf{J} \text { (variable; } \\
\text { statistic) }\end{array}$ & K (variable; statistic) \\
\hline $\begin{array}{l}\text { Marital status } \\
\text { Single } \\
\text { Married } \\
\text { Divorced } \\
\text { Widowed }\end{array}$ & $\begin{array}{c}\mathrm{r}=-0.112 \\
\mathrm{p}=0.083\end{array}$ & $\begin{array}{l}r=-0.157 \\
p=0.013^{*}\end{array}$ & $\begin{array}{l}r=-0.058 \\
p=0.366\end{array}$ & $\begin{array}{c}r=-0.039 \\
p=0.544\end{array}$ & $\begin{array}{l}r=-0.164 \\
p=0.010^{*}\end{array}$ \\
\hline $\begin{array}{l}\text { Socio-economic } \\
\text { Class } 1 \\
\text { Class } 2 \\
\text { Class } 3 \\
\text { Class } 4 \\
\text { Class } 5\end{array}$ & $\begin{array}{l}\mathrm{r}=-0.456 \\
\mathrm{p}=0.000^{*}\end{array}$ & $\begin{array}{l}\mathrm{r}=-0.432 \\
\mathrm{p}=0.000^{*}\end{array}$ & $\begin{array}{l}r=-0.385 \\
p=0.000 *\end{array}$ & $\begin{array}{l}\mathrm{r}=-0.351 \\
\mathrm{p}=0.000^{*}\end{array}$ & $\begin{array}{l}\mathrm{r}=-0.342 \\
\mathrm{p}=0.000^{*}\end{array}$ \\
\hline $\begin{array}{l}\text { Household size } \\
1-4 \\
5-7 \\
>7\end{array}$ & $\begin{array}{l}\mathrm{r}=0.113 \\
\mathrm{p}=0.078\end{array}$ & $\begin{array}{l}r=0.175 ; \\
p=0.006^{*}\end{array}$ & $\begin{array}{l}r=0.003 \\
p=0.962\end{array}$ & $\begin{array}{l}\mathrm{r}=0.070 \\
\mathrm{p}=0.269\end{array}$ & $\begin{array}{l}\mathrm{r}=0.159 ; \\
\mathrm{p}=0.013^{*}\end{array}$ \\
\hline $\begin{array}{l}\text { Household inco } \\
<50,000 \\
50,000-100,000 \\
>100,000\end{array}$ & $\begin{array}{l}\mathrm{r}=0.299 \\
\mathrm{p}=0.000^{*}\end{array}$ & $\begin{array}{l}\mathrm{r}=0.288 \\
\mathrm{p}=0.000^{*}\end{array}$ & $\begin{array}{l}\mathrm{r}=0.242 ; \\
\mathrm{p}=0.000^{*}\end{array}$ & $\begin{array}{l}\mathrm{r}=0.205 ; \\
\mathrm{p}=0.001 *\end{array}$ & $\begin{array}{l}\mathrm{r}=0.188 \\
\mathrm{p}=0.003^{*}\end{array}$ \\
\hline
\end{tabular}

*statistically significant $(\mathrm{p}<0.05)$

$\mathrm{G}=$ Adults in household skip meals or cut size of meal; $\mathrm{H}=$ eat less than you feel you should; I=hungry but no food; $\mathrm{J}=$ lose weight due to insufficient food; $\mathrm{K}=$ Adults in household go without food for a day because no money 
Table 6. Distribution of food security measures of households by selected socio-demographic characteristics contd

\begin{tabular}{|c|c|c|c|c|c|}
\hline Variable & $\begin{array}{l}\text { L( statistic; } \\
\text { p value) }\end{array}$ & $\begin{array}{l}\text { M( statistic; } \\
\text { p value) }\end{array}$ & $\begin{array}{c}\text { N (statistic; } \\
\text { p value) }\end{array}$ & $\begin{array}{l}\text { O (statistic; } \\
\text { p value) }\end{array}$ & $\begin{array}{c}\text { P (statistic; } \\
\text { p value) }\end{array}$ \\
\hline $\begin{array}{c}\text { Marital status } \\
\text { Single } \\
\text { Married } \\
\text { Divorced } \\
\text { Widowed }\end{array}$ & $\begin{array}{l}\mathrm{r}=0.009 \\
\mathrm{p}=0.885\end{array}$ & $\begin{array}{c}\mathrm{r}=-0.101 \\
\mathrm{p}=0.102\end{array}$ & $\begin{array}{c}\mathrm{r}=-0.030 \\
\mathrm{p}=0.644\end{array}$ & $\begin{array}{l}\mathrm{r}=-0.141 ; \\
\mathrm{p}=0.031^{*}\end{array}$ & $\begin{array}{c}\mathrm{r}=-0.045 \\
\mathrm{p}=0.492\end{array}$ \\
\hline $\begin{array}{l}\text { Household size } \\
\qquad \begin{array}{c}1-4 \\
5-7 \\
>7\end{array}\end{array}$ & $\begin{array}{l}\mathrm{r}=0.041 \\
\mathrm{p}=0.534\end{array}$ & $\begin{array}{l}\mathrm{r}=0.039 ; \\
\mathrm{p}=0.547\end{array}$ & $\begin{array}{c}\mathrm{r}=-0.036 \\
\mathrm{p}=0.580\end{array}$ & $\begin{array}{c}\mathrm{r}=-0.112 \\
\mathrm{p}=0.086\end{array}$ & $\begin{array}{c}\mathrm{r}=-0.102 \\
\mathrm{p}=0.117\end{array}$ \\
\hline $\begin{array}{c}\text { Household income } \\
<50,000 \\
50,000-100,000 \\
>100,000\end{array}$ & $\begin{array}{l}\mathrm{r}=0.213 ; \\
\mathrm{p}=0.001 *\end{array}$ & $\begin{array}{l}\mathrm{r}=0.156 ; \\
\mathrm{p}=0.016^{*}\end{array}$ & $\begin{array}{l}\mathrm{r}=0.250 \\
\mathrm{p}=0.000^{*}\end{array}$ & $\begin{array}{l}\mathrm{r}=0.167 \\
\mathrm{p}=0.010^{*}\end{array}$ & $\begin{array}{l}r=0.186 \\
p=0.004^{*}\end{array}$ \\
\hline
\end{tabular}

*statistically significant $(\mathrm{p}<0.05)$

$\mathrm{L}=$ how often did you or adults in household go a day without food; M=Ever cut size of children's meal because no money; N=Children ever skip meal due to insufficient food; $\mathrm{O}=$ Children go without food for a whole day; $\mathrm{P}=$ Children ever hungry but you can't afford more food.

\section{Discussion}

\subsection{Food Security Status of Respondents}

Food insecurity is indeed a major problem in Uyo as shown by this study, where almost nine in ten households were food insecure with about a third experiencing severe hunger. This is in agreement with findings from other studies [21,23]. A possible explanation to the high prevalence of food insecurity is the fact that Akwa Ibom State is predominantly a civil service state [25]; this means that the public sector employs a significant proportion of the labor force. Therefore, any delay, reduction or lack of payment of salaries by the government results in a catastrophic chain reaction. For example, the civil servants will lack adequate funds to buy goods from the market women, who will in turn lack adequate funds to buy more foodstuffs. This results in a widespread reduction in household income which would in turn affect the household food security status.

\subsection{Relationship between Socio-demographic Characteristics of Respondents and Household Food Security}

The socio-demographic characteristics of individuals significantly influence their food security status. With respect to marital status, there was a positive relationship between being married and household food insecurity and this finding agrees with a previous finding [10]. This may be because married household heads may have a larger household to cater for, and household size has an inverse relationship with food security [26]. This picture was seen in this study as those with the largest household sizes were the most food insecure with severe hunger. Also, over half of widowed households suffered from severe hunger. This was not surprising as the surviving household heads would have no support as they become the sole breadwinners of often large households.

In this study, higher socioeconomic classes and higher household incomes had positive associations with food security. This is in agreement with findings of other studies [10,26,27]. This is expected as household income determines how much can be spent on various needs of the household. This finding is in agreement with Sekhampu (2013) who reported that household income was positively associated with food security status of the respondents [26].

\subsection{Coping Strategies of Respondents}

This study also analyzed the compensatory feeding mechanisms/coping strategies engaged in by respondents when money was insufficient for food. The prevailing strategies of borrowing money and collecting food from friends, neighbors and relatives supported findings of other studies [28,29,30]. These coping strategies serve deleterious purposes, further worsening food insecurity as families who borrow are plunged into debt which they have to pay with their next income. This produces a vicious cycle of food insecurity and debt. Another coping strategy employed by the respondents was sending children to work. Child labor is common in the study area, the commonest type being street hawking. These children are then exposed to numerous health problems, road traffic accidents, physical and sexual abuse, robbery and street fights. Also, these children perform poorly in school [31]. Other coping strategies including starving to feed the children, sending the children to beg for food or money and sending children to stay in a relative's or friend's house were also employed by the households. Sending 
children to beg for food/money is not a common practice in the southern part of the country as opposed to the North, where children make up majority of street beggars [32]. Food insecurity is a real situation in the study area which must be tackled accordingly.

\section{Conclusion}

This study, which assessed the household food security among different wealth groups within Uyo metropolis, showed that most of the respondents were food insecure. Low socioeconomic status, low household income and large household size were associated with a higher level of food insecurity. When funds were insufficient for food, majority of the respondents either borrowed money or collected food from friends, neighbors and relatives, and some sent children to work. These coping strategies cause a great burden on households, further worsening their food insecurity. It is therefore recommended that better educational opportunities be made available and accessible to those of the low socioeconomic class, so as to ensure gainful employment. The minimum wage of the country should be increased, which will result in increased household income. Public health interventions on family planning and benefits of smaller household sizes should also be encouraged.

\section{References}

[1] FAO/WHO. Proceedings of the international conference on nutrition. Rome, 1992.

[2] FAO. Rome declaration on food security and world food summit plan of action: the state of food insecurity in the world. Rome, 1996.

[3] Adekoya A. E. Food insecurity and coping strategies among rural households in Oyo state, Nigeria. Journal of food agriculture and environment; 7(3 \&4): 187-191, 2009.

[4] Bickel, G., Nord, M., Price, C., Hamilton, W and Cook, J. Measuring food security in the United States: guide to measuring household food security, Alexandria, VA, US department of agriculture, 2000.

[5] Max R. and Hannah R. Hunger and undernourishment. Published online at ourworldindata.org. 2018. Retrieved from https://ourworldindata.org/hunger-and-undernourishment

[6] Ubokudom O. O., Namso N. F., Egbe B. E., kesit k. N. Household food security status and its determinants among rural farmers in Akwa Ibom state Nigeria. Agricultural Science Research Journal ;7 (10): 297-303, 2017

[7] Arene C. J. and Anyaeji R. C. Determinants of food security among households in Nsukka metropolis of Enugu state, Nigeria. Pakistan journal of social sciences; 30(1): 9-116, 2010.

[8] The World Bank. Poverty. 2018. Retrieved from www.worldbank.org/en/topic/poverty/overview

[9] Oyebanjo, O., Ambali, O. I and Akerele, E. O. Determinants of food security status and incidence of food insecurity among rural farming households in Ijebu division of Ogun state Nigeria. Journal of agricultural science and environment; 13: 92-103, 2013.

[10] Adepoju, A. O. and Adejare, F. E. Food insecurity status of rural households during the post-planting season in Nigeria. Journal of agriculture and sustainability; 4 (1): 16-35, 2013.

[11] Mensah E, Lal A, Guerrero B. L. and Lust D. G. Agriculture and the state of food insecurity in Western Africa. Selected paper presentation at Southern agricultural economics association $48^{\text {th }}$ annual meeting, San Antonio Texas, 2016.

[12] Akinyele, I. O. Ensuring food and nutrition security in rural Nigeria; an assessment of the challenges, information needs and analytical capacity. Nigeria strategy support program (NSSP) background paper no nssp 007. International research institute Washington DC, 2009.

[13] Etim P. Towards achieving food security in Akwa Ibom state. Hyperstation. ,2015. Retrieved from www.hyperstationng.com/promise-etim-towards-achieving-foodsecurity-in-akwa-ibom-state/.

[14] Fakayode, S. B., Rahji, M. A., Oni, O. A. and Adeyemi, M. O. An assessment of food security situations of farm households in Nigeria: a USDA approach. The social sciences; 4(1): 24-29, 2009.

[15] Global hunger index. Global hunger index by severity, 2017. Retrieved from http://www.globalhunger.org/result-2017.

[16] Ajani, S. R., Badejo, C. A. and Yusuf, B. O.Measuring household food insecurity in selected local governmentareas of Lagos and Ibadan, Nigeria. Pakistan Journal of Nutrition; 5 (1): 62-67, 2006.

[17] Omotesho, O. A. and Muhammad-Lawal, A. Optimal food plan for rural households' food security in Kwara state, Nigeria: the goal programming approach. Journal of agricultural biotechnology and sustainable development; 2(1):7-14, 2010.

[18] Jyoti, D. F., Frongillo, E. A. and Jones, S. J. Food insecurity affects school children's academic performance, weight gain, and social skills. Journal of nutrition; 135(12): 2831-283, 2005.

[19] Stuff, J. E., Casey, P. H., Szeto, K. L., Gossett, J. M., Robbins, J. M., and Simpson, P. M., et al. Household food insecurity is associated with adult health status. Journal of nutrition; 134(9): 2330-2335, 2004.

[20] Matheson, D. M., Varady, J., Varady, A and Killen, J. D. Household food security and nutritional status of Hispanic children in the fifth grade. Journal of Clinical Nutrition; 76(1): 210-217, 2002.

[21] Ibok O. W., Idiong C. I., Itoro N. B., Iniobong I. O., and Uwemedimo E. O. Analysis of food insecurity status of urban food crop farming households in cross river state Nigeria: a USDA approach. Journal of agricultural science; 6(2): 201-204, 2014.

[22] Ekpenyong R. E. An assessment of the threats to food security in Akwa Ibom state, Nigeria, 2016. Retrieved from

https://www.researchgate.net/publication/228858092_An_assessm ent_of_the_threats_to_food_security_in_Akwa_ibom_state_Niger ia.

[23] Opara D. C. and Madukosiri H. C. Food security and outlook on life by undergraduates and post graduate students of the University of Uyo. Journal of nursing and health science; 5(5) version 11: 71-80, 2016.

[24] Brinkhoff, T. Akwa Ibom State in Nigeria. City populationstatistics, maps and charts, 2017. Retrieved from https://www.citypopulation.de/php/nigeriaadmin.php?adm1id=NGA003.

[25] Nigeria galleria. Business economy of Akwa Ibom state, 2017. Retrieved from

https://www.nigeriagalleria.com/Nigeria/states_Nigeria/akwaibom/business-economy-akwa-ibom-state.html.

[26] Sekhampu, T. Determination of factors affecting the food security status of households in Bophelong, South Africa. International Business and Economics Research Journal (IBER; 12(5): 543-550. 2013.

[27] Nyako, A.M. The relationship between educational attainment and food security in Nigeria. Digitalgeorgetown, 2013. Retrieved from http://hdl.handle.net/10822/558648.

[28] Ezeama N. N., Christian I., Adinma E., Emelumadu O., and Adogu P. Coping with household food insecurity: perspectives of mothers in Anambra State, Nigeria. Journal of food security; 3(6): 145-54, 2015.

[29] Shuaibu H., Abdullahi A., Ango A. K., Yusuf H. A., Sulaiman R., and Bello M. (2015). Food security and coping strategies of selected villages along river Rima affected by flood in Sokoto state, Nigeria. Asian Journal of Agricultural Extension, Economics and Sociology; 6(2):77-84, 2015.

[30] Akerele, D., Momoh, S., Aromolaran A., and Shittu A.. Food insecurity and coping strategies in the South-west in Nigeria. Food Security; 5(3): 407-414, 2013

[31] Johnson O. E. and Ihesie C. A. Social implications and factors associated with street hawking among children in Uyo, Akwa Ibom State, Nigeria. British Journal of Education, Society and Behavioral Science. 11(2):1-9, 2015.

[32] Bukoye R. O. Prevalence and consequences of street begging among adults and children in Nigeria, Suleja metropolis. Procedia-Social and Behavioral Sciences; 171: 323-333, 2015. 